TECHNICAL NOTES AND SHORT PAPERS

\title{
Characteristic Exponents of Mathieu Functions
}

\author{
By T. Tamir
}

The study of the properties and solutions of the Mathieu second-order differential equation has been very extensive in the last decades, due to the special interest presented by physical problems involving periodic media and problems separable in elliptic coordinate systems, [1], [2]. The purely periodic solutions of the Mathieu equation have been extensively tabulated with high accuracies over large ranges of the parameters involved [3], [4].

On the other hand, numerical tables for the non-periodic Floquet-type solutions are scarce [5], [6], [7]; in addition, the increments between two successive tabulated values are relatively large for some of these tables and the accuracy is relatively low in the other tables. Consequently, the applicability of the available data is rather limited.

The regions of "stable" solutions of the Mathieu equation are of particular interest in applications involving the wave equation with periodic boundary conditions, since these regions correspond to the propagating waves in the corresponding media. The first three regions of stable solutions were tabulated with this type of problem in mind, the numerical results being given in Tables I, II, and III.

The canonical form of the Mathieu equation considered is given by

$$
y^{\prime \prime}+(p-2 q \cos 2 z) y=0,
$$

with $p$ and $q$ real. Every non-periodic solution of (1) is a linear combination of two linearly independent Floquet-type solutions of the form

$$
y=e^{i v z} P(z),
$$

where $P(z)$ is a periodic function of $z$, and $\nu$ is the so-called cbaracteristic exponent. This exponent is a function of the parameters $p$ and $q$ only. When $\nu$ is real, the solutions (2) are called "stable" since they are uniformly bounded for all real $z$; then $\nu=f(p, q)$ may be chosen to be single-valued. (Note: In the tables and curves shown here, $\nu$ is not reduced to $0 \leqq \nu \leqq 1$ as is usually done, but its actual value is taken, thus defining the particular stable region it represents.)

It is shown in the references that all values of $\nu$ are solutions of the continued fraction equation

$$
D_{0}=\frac{1 \mid}{D_{1}}-\frac{1 \mid}{\mid D_{2}}-\frac{1 \mid}{\mid D_{3}}-\cdots+\frac{1 \mid}{D_{-1}}-\frac{1 \mid}{\mid D_{-2}}-\frac{1 \mid}{\mid D_{-3}}-\cdots
$$

where

$$
D_{m}=\frac{(2 m+\nu)^{2}-p}{q}, \quad(m=0, \pm 1, \pm 2 \cdots) .
$$

Received June 12, 1961. The work described in this paper was supported by the Air Force Cambridge Research Laboratories. 
For computation purposes, it is convenient to define

$$
x=\nu^{2}-p
$$

so that equation (3) will take the form

$$
\begin{aligned}
& x=\frac{q^{2}}{4(1+\nu)+x}-\frac{q^{2} \mid}{\mid 8(2+\nu)+x}-\cdots-\frac{q^{2}}{\mid 4 n(n+\nu)+x}-\cdots \\
& +\frac{q^{2} \mid}{4(1-\nu)+x}-\frac{q^{2} \mid}{\mid 8(2-\nu)+x}-\cdots-\frac{q^{2}}{\mid 4 n(n-\nu)+x}-\cdots \\
& \quad(n=1,2,3, \cdots)
\end{aligned}
$$

The continued fractions are convergent since they satisfy the convergence test [2] which requires that $\left|D_{n}\right| \geqq 2$ for $n>N$, where $N$ is a finite integer.

To find the roots of equation (6), it is noted that this equation is already expressed in a suitable form for solution by means of an iteration process, with $x$ regarded as the unknown. Taking $q$ and $\nu$ as variable parameters, such an iteration procedure was programmed on a computer which obtained $p$ with an accuracy of $10^{-5}$. As is proved in the Appendix, the iteration process converges in an alternating fashion, i.e., the exact result always lies between the values obtained by two successive iterations; hence, the iteration process must be carried out only until the difference between two successive results is less than the maximum desired deviation.

It was also noted that the number of iterations required to solve equation (6) was extremely large for a large range of parameters if a simple iteration procedure was used. This was due to a very slow convergence of the iteration process in some regions of $q$ and $\nu$. To improve this situation, the computer was programmed to differentiate between two cases:

a) "Fast" convergence: $x_{n+1}^{\prime}$ contained within the interval $\left(x_{n}, x_{n+2}\right)$,

b) "Slow" convergence: $x_{n+1}^{\prime}$ outside the interval $\left(x_{n}, x_{n+2}\right)$, where $x_{n+1}$ is the result of the $n$th iteration, using $x_{n}$ as the trial value; $x_{n+1}^{\prime}$ is the arithmetic mean of $x_{n+1}$ and $x_{n}$.

After using the test to determine whether the convergence is "fast" or "slow," as defined above, the computer used either $x_{n+2}$ or $x_{n+2}^{\prime}$, respectively, as the next trial value for the $n+2$ iteration. This procedure reduced the number of iterations required from more than a thousand to less than twenty for the most slowly converging cases.

The results thus obtained are given in the appended tables, while Figure 1 shows these values plotted in the $p-q$ plane. Note that, for greater clarity, $P= \pm \sqrt{{ }^{\mid} p^{1}}$ rather than $p$ is plotted in the figure. Thus $p=P^{2}$ if $P \geqq 0 ; p=-P^{2}$ if $P<0$.

Appendix. It is shown below that an iteration process based on equation (6), if converging, will do so in an alternating manner; i.e., the value of the exact solution always lies between the values of the results of any two successive iterations.

The iteration process may be written in the form

$$
x_{n+1}=R_{0}^{+}\left(x_{n}\right)+R_{0}^{-}\left(x_{n}\right)
$$




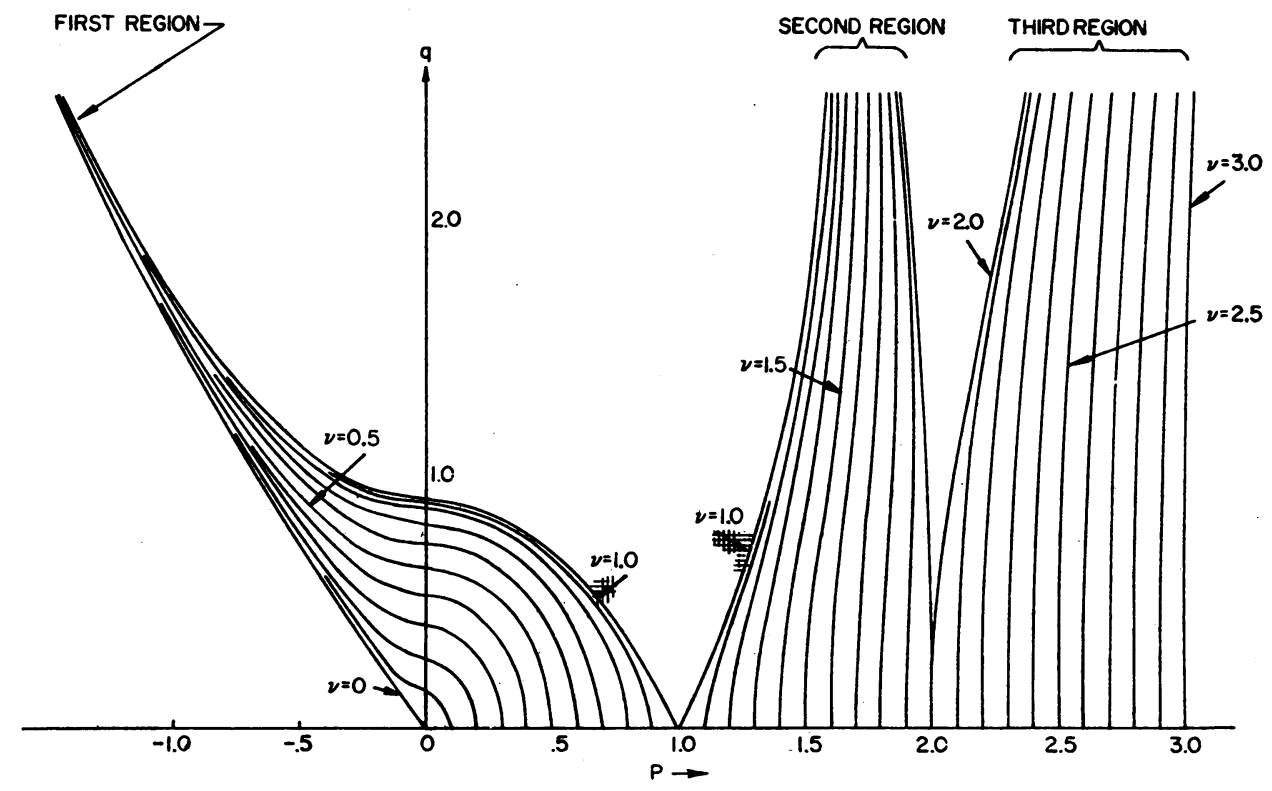

Fig. 1-Curves of $q$ vs $P$ for constant values of $\nu$. The values of $\nu$ pertaining to each curve are given directly by the interceptions with the $P$ axis. $p=P^{2}$ if $P \geqq 0 ; p=-P^{2}$ if $P<0$.

where

$$
R_{0}^{ \pm}\left(x_{n}\right)=\frac{1}{a_{0}^{ \pm} x_{n}+b_{0}^{ \pm}}-\frac{1}{! a_{1}^{ \pm} x_{n}+b_{1}^{ \pm}}-\cdots-\frac{1}{\mid a_{m}^{ \pm} x_{n}+b_{m}^{ \pm}}-\cdots .
$$

Then

$$
R_{m}^{ \pm}\left(x_{n}\right)=\frac{1}{a_{m}^{ \pm} x_{n}+b_{m}^{ \pm}}-\frac{1}{\mid a_{m+1}^{ \pm} x_{n}+b_{m+1}^{ \pm}}-\cdots
$$

is a "remainder" function or the "tail" of a continued fraction after the first $m$ terms have been omitted.

Disregarding the \pm sign, we have

$$
R_{0}\left(x_{n}\right)=\frac{1}{a_{0} x_{n}+b_{0}-R_{1}\left(x_{n}\right)} .
$$

Differentiating with respect to $x_{n}$, we obtain

$$
R_{0}^{\prime}\left(x_{n}\right)=-\frac{a_{0}-R_{1}^{\prime}\left(x_{n}\right)}{\left[a_{1} x_{n}+b_{1}-R_{1}\left(x_{n}\right)\right]^{2}}=-R_{0}^{2}\left[a_{0}-R_{1}^{\prime}\left(x_{n}\right)\right] .
$$

Noting that $R_{m}\left(x_{n}\right)$ has the same functional form as $R_{0}\left(x_{n}\right)$, one finds

$$
R_{0}^{\prime}\left(x_{n}\right)=-\left[a_{0} R_{0}^{2}+a_{1}\left(R_{0} R_{1}\right)^{2}+a_{2}\left(R_{0} R_{1} R_{2}\right)^{2}+\cdots\right]
$$

In the case considered, $a_{m}=\frac{1}{q^{2}}$ or 1 ; hence $a_{m}>0 . R_{m}\left(x_{n}\right) \rightarrow 0$ as $m \rightarrow \infty$ so that $R_{0}^{\prime}\left(x_{n}\right)$ has a finite negative value for any finite $x_{n}$. Consequently, the derivative of $x_{n+1}$ is finite and negative so that the iteration process based on equation (6), if convergent, will converge in an alternating fashion. 


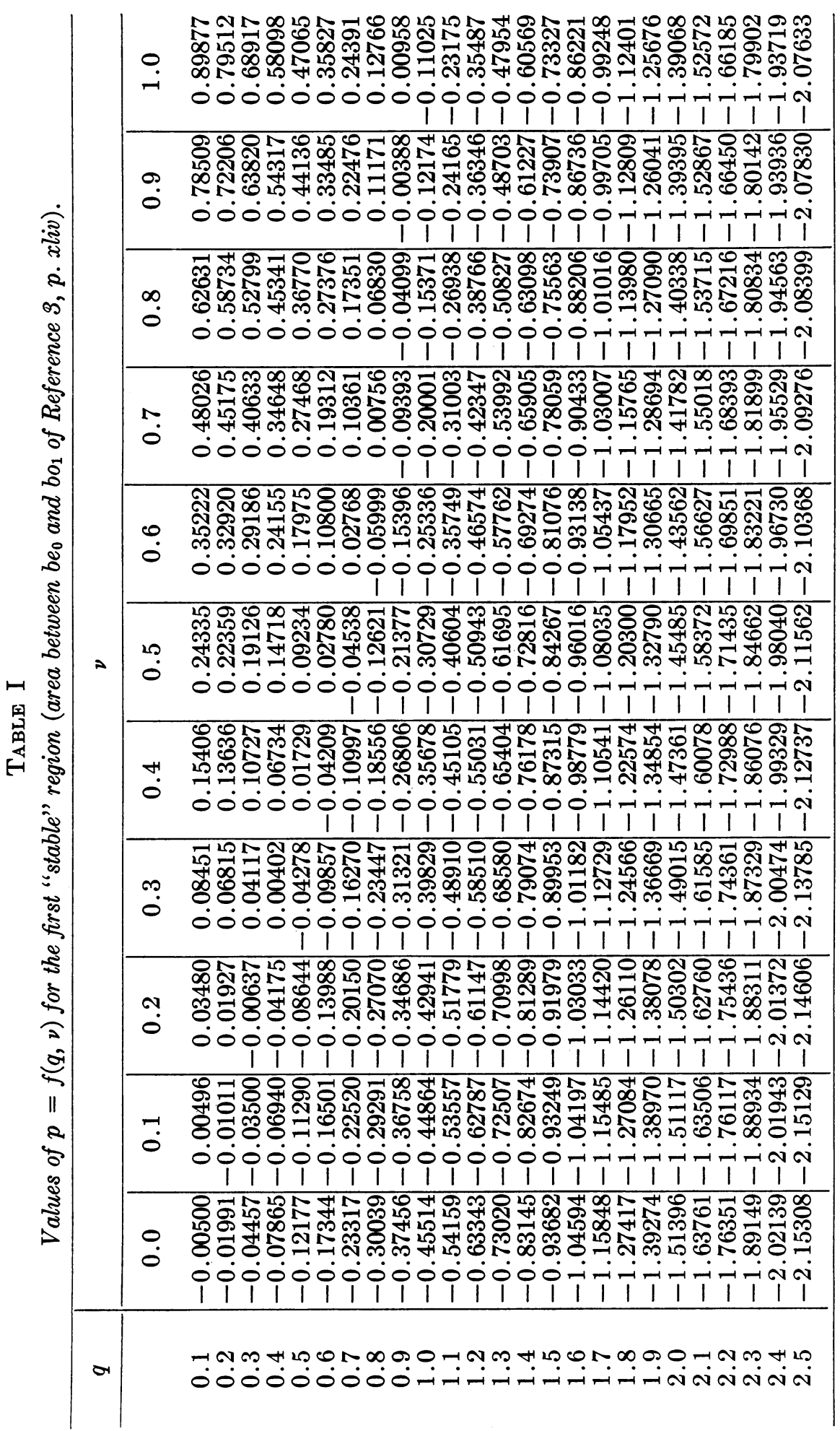




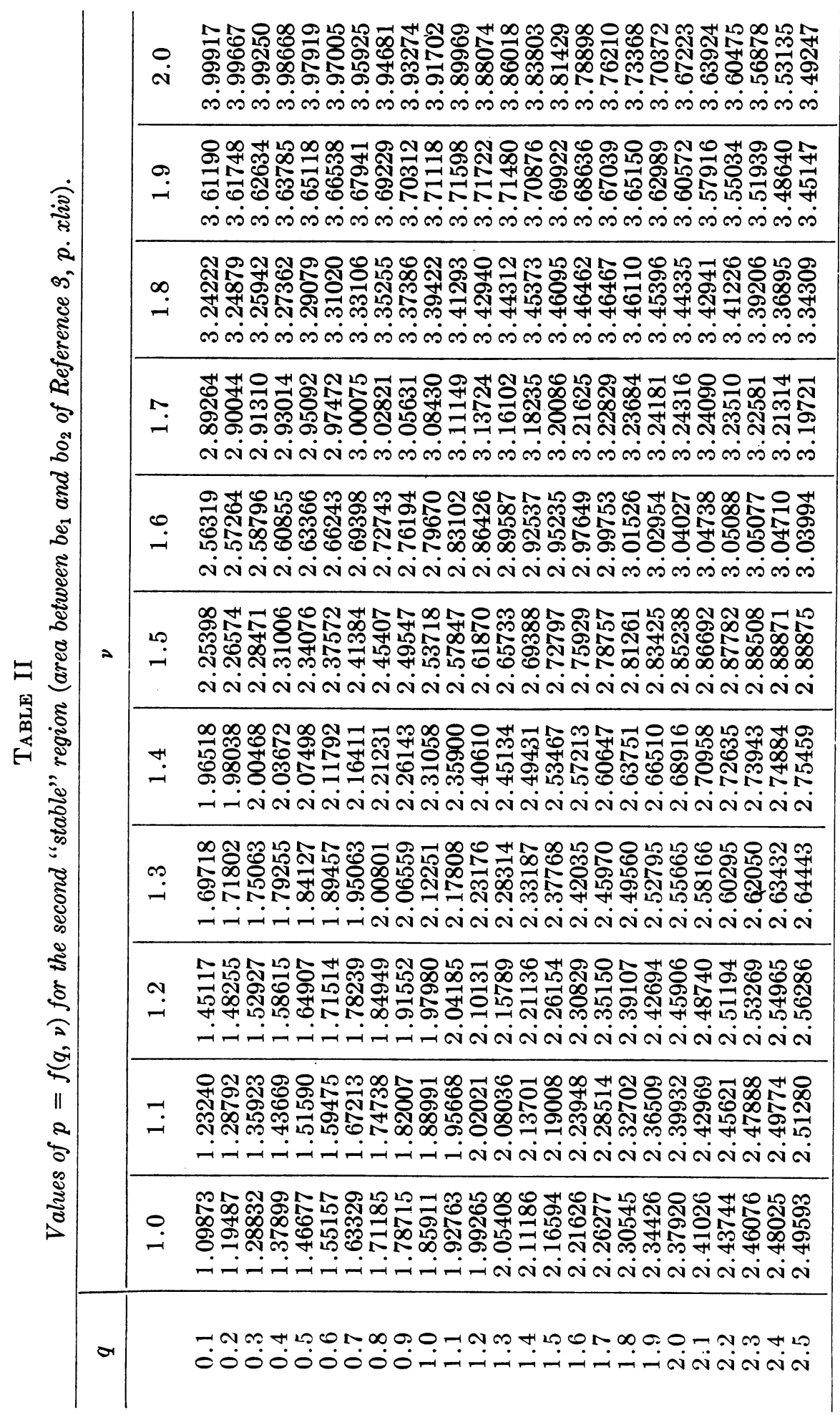




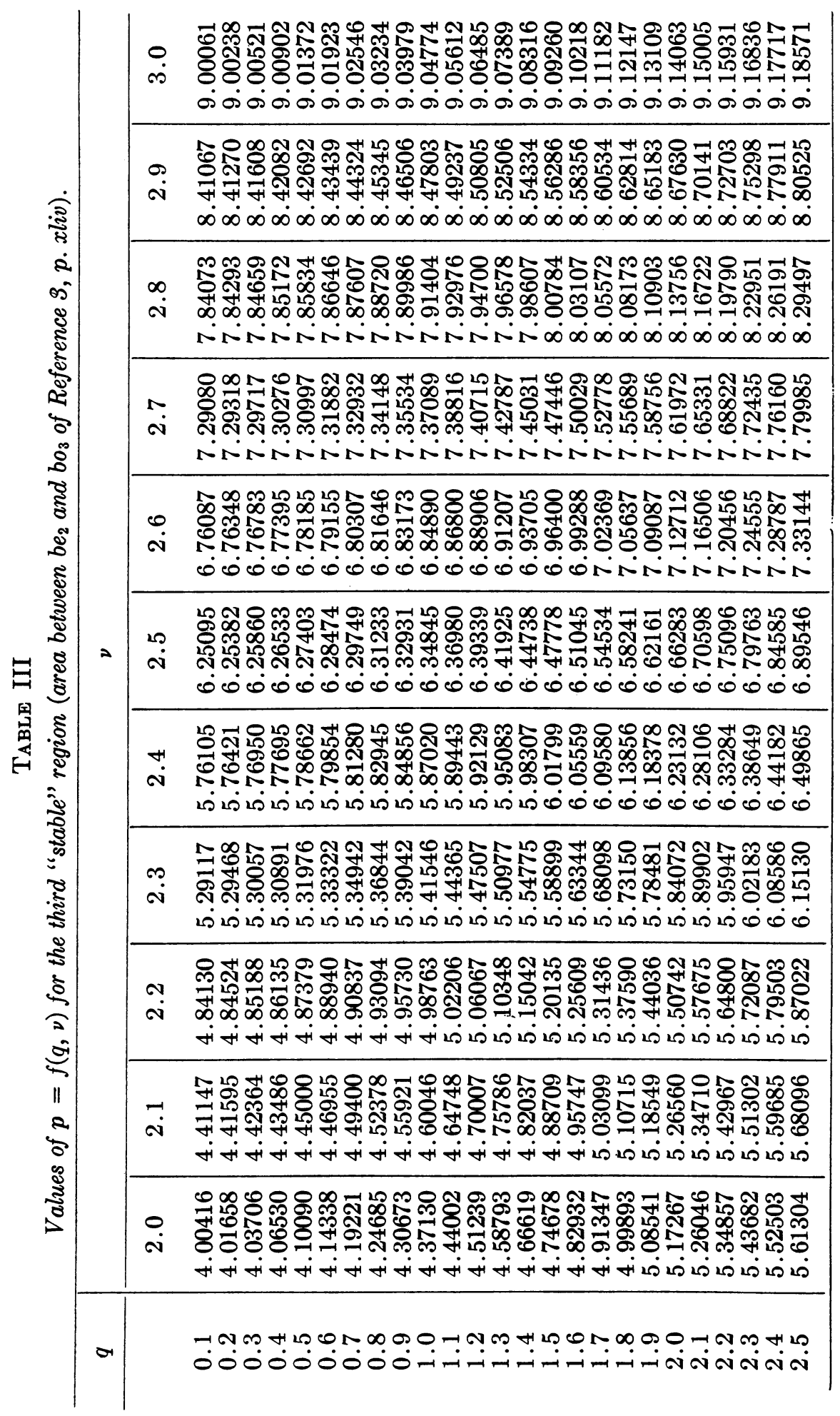


No absolute convergence proof was found for the iteration process itself; on the other hand, no convergence instabilities were experienced in the actual computation for the range of parameters that was tabulated.

Acknowledgement. The author wishes to express his indebtedness to Professor A. A. Oliner for his interest and help in connection with this paper, and to Dr. Gertrude Blanch, Aeronautical Research Laboratory, for providing valuable references and information. Special thanks are due to John Segal for his patience and ingenuity in carrying out the programming and obtaining the numerical results.

Microwave Research Institute

Polytechnic Institute of Brooklyn

Brooklyn, New York

1. N. W. Mclachlan, Theory and Application of Mathieu Functions, University Press, Oxford, 1951.

2. J. MeIXner \& F. W. Schärke, Mathieusche Funktionen und Sphäroidfunktionen, Springer-Verlag, Berlin, 1954 .

3. Nat. BUR. STandard, Tables Relating to Mathieu Functions, Columbia University Press, New York, 1951.

4. J. C. WILTSE \& M. J. KING, Values of Mathieu Functions, Johns Hopkins Radiation Laboratory, Technical Report No. AF-53, 1958.

5. J. G. BRaINERd \& C. N. Weygand, "Solutions of Mathieu's equations," Phil. Mag., v. 30,1940, p. $458-477$.

6. H. J. Gray, R. Merwin \& J. G. Brainerd, "Solution of the Mathieu equation," Amer. Inst. Electr. Engrs., v. 67, 1948, p. 429-441.

7. S. J. ZAROODNY, An Elementary Review of the Mathieu-Hill Equation of Real Variables Based on Numerical Solutions, Ballistic Research Laboratories, Aberdeen Proving Ground Maryland, Memo. Rep. No. 878, April 1955.

\title{
An Algorithm for Solving Certain Polynomial Equations with Coefficient Parameters
}

\author{
By Robert D. Stalley
}

1. Introduction and General Method. We describe a storage-saving procedure that can be used in real time simulation or other situations in which the roots of an equation of the form

$$
\begin{aligned}
\sum_{j=0}^{n} F_{j}\left(x_{1}, x_{2}, \cdots, x_{m}\right) y^{e_{j}}=0, \quad m \geqq 2, \quad 0 \leqq e_{0} \leqq e_{1} \leqq & \cdots \leqq e_{n}, \\
& e_{j} \text { integral }(j=0,1, \cdots, n),
\end{aligned}
$$

must be obtained within severe time limits, i.e., from storage, for values of the coefficient parameter $m$-tuple $\left(x_{1}, x_{2}, \cdots, x_{m}\right)$ from some given set. The exponents $e_{j}$ are defined so as to be monotonically increasing rather than strictly increasing for later notational convenience.

Suppose there exist relations

$$
u_{i}=\varphi_{i}\left(x_{1}, x_{2}, \cdots, x_{m}\right), \quad(i=\mu, \mu+1, \cdots, m), 2 \leqq \mu \leqq m,
$$

Received February 21, 1961. 